\title{
CHALLENGES, CLASSIFICATION AND COMPARATIVE STUDY OF QOS SOLUTIONS IN MANET
}

\author{
Neelam Phate, M.A. Rizvi \\ Dept. of CEA, NITTTR Bhopal, Bhopal, India \\ neelamphate@gmail.com \\ Dept. of CEA, NITTTR Bhopal, Bhopal, India \\ marizvi@nitttrbpl.ac.in
}

\begin{abstract}
A mobile ad-hoc network (MANET) is composed of mobile nodes without any fixed infrastructure. Mobile nodes are communicating with each other over radio links. The large amount of real-time traffic involves high bandwidth and liable to congestion. Routing in MANET is a challenging task due to high mobility of nodes in a network. Quality of Service (QoS) is an important consideration in MANET. It is more difficult to guarantee QoS in MANETs than in other type of networks. To establish the routes and to secure the resources necessary to provide the QoS requires collaboration among the nodes. Therefore, QoS routing protocols face the challenge of delivering data to destinations. The appropriate QoS metrics should be used, such as bandwidth utilization, delay, packet delivery ratio (PDR) and jitter for QoS based routing. This paper provides the challenges, classification and comparison of some of the QoS solutions in MANET.
\end{abstract}

\section{Keywords}

MANET; MANET issues; MANET challenges; QoS; Routing Protocol.

\section{Council for Innovative Research}

Peer Review Research Publishing System

\section{Journal: INTERNATION JOURNAL OF COMPUTERS AND TECHNOLOGY}

Vol. 13 , No. 6

editorijctonline@gmail.com

www.cirworld.org/journals 


\section{ISSUES AND CHALLENGES OF QOS BASED ROUTING PROTOCOL}

Regardless of the attractive applications, the features of MANET introduce several challenges that must be studied carefully before a wide commercial deployment can be expected. These includes:

1. Dynamic topology: In MANET nodes are mobile that leads to changing the topology dynamically and most likely to fail of a path.

2. Limited resource availability: Battery life, bandwidth, memory, processing capability and other resources in MANET affect the QoS aware routing.

3. Variable capacity links: The availability of bandwidth in MANETs is scarce [1] and the main problem is the estimation of bandwidth because it varies with the mobility of nodes.

4. Energy constrained operation: The mobile nodes are constrained by limited power supply as they rely on batteries for their energy.

5. Lack of centralized control: No central controller to coordinate the activities of mobile nodes. This complicates the QoS provisioning mechanism in MANET.

6. Maintenance of route: Due to dynamic topology in MANET, it is difficult to achieve a fast route maintenance scheme into QoS-aware routing.

7. Security: MANETs is more prone to security attacks such as eavesdropping, spoofing and denial of service and this is an important QoS constraint in military application.

8. Error-prone channel: Radio waves in wireless medium suffer from interference, attenuation and multipath propagation because of its broadcast nature.

9. Hidden and Exposed terminal problem: Hidden terminals are hidden nodes to the sender, but are reachable to the receiver. It reduces the throughput of the QoS aware routing protocol. Exposed terminals are nodes in the transmission of the sender, but prevented from making a transmission.

\section{CRITICAL PARAMETER FOR QOS IN MANET}

1. Throughput: It is defined as the maximum fraction of channel bandwidth used by successfully transmitted packets [2].

2. Delay: It is defined as the time taken by a packet to travel from the source to the destination. Overall end-to-end delay is calculated as:

[End-to-end delay = Propagation delay + Transmission delay + Packetizations delay + Congestion delay + Access delay + Queuing delay]

3. Jitter: It is defined as the variation in the delay of packets at the destination. Some time-sensitive applications may become affected by jitter.

4. Packet Delivery Ratio: It is defined as the ratio of the packets received successfully to the packets sent. It is calculated as follows:

$$
P D R=\frac{\text { No. of packets recieved }}{\text { No. of packets sent }}
$$

5. Scalability: It is defined as the ability of ad hoc networks to continue to function well when the number of nodes increases.

6. Energy: Energy efficient routing protocols enhancing the lifetime of the network by bringing off the sources and consumers energy in a node and in a network.

7. Security: It is important in military applications, especially in MANET because there is no central control and shared wireless medium.

\section{CLASSIFICATION OF QOS SOLUTIONS}

QoS solutions are classified into several categories based on the mechanisms and techniques used. Classification and its sub-classification based on diverse categories are as follows:

\section{A. Classification based on QoS approach}

1) Based on interaction between routing protocol and QoS provisioning mechanism

- $\quad$ Core Extraction Distributed Ad Hoc Routing protocol (CEDAR) [3]: It is oriented to small and middle size networks. Route computation is on demand, and is performed by the core nodes. It consists of three main components:

Core extraction: A set of nodes is distributively and dynamically selected to form the core, which maintains local topology and performs route calculations.

- Link state propagation: propagating bandwidth availability information of stable high bandwidth connections to all core nodes, while information of dynamic links or low bandwidth is kept local.

- QoS Route Computation: A core path is established first from dominator (neighboring core node) of the source to dominator of destination. Using up-to-date local topology, dominator of source finds a path satisfying the requested 
QoS from source to furthest possible core node. This furthest core node, then becomes the source of the next iteration. The above process repeats until destination is reached or the computation fails to find a feasible path.

The advantage of this protocol is utilization of core nodes reduces the traffic overhead. Disadvantages of CEDAR is that it finds Sub-optimal route and the route establishment and computation is relied on core nodes hence core nodes being bottlenecked. Core node movement affects the performance of the protocol.

- QoS AODV [4]: QoS for AODV was proposed by C. Perkins and E. Royer. When a node in AODV desires to send a message to some destination node, it initiates a Route Discovery Process (RREQ). The main idea of making AODV QoS enabled is to add extensions to the route messages (RREQ, RREP). A node that receives a RREQ + QoS Extension must be able to meet the service requirement in order to rebroadcast the RREQ (if not in cache). In order to handle the QoS extensions some changes need to be on the routing tables

AODV current fields:

Destination Sequence Number, Interface, Hop Count, Next Hop, List of Precursors

- QoS AODV fields:

1) Maximum Delay, 2) Minimum Available Bandwidth, 3) List of Sources Requesting Delay Guarantees and 4) List of Sources Requesting Bandwidth Guarantees

2) Based on interaction between network and MAC layers:

- $\quad$ Ticket Based QoS Routing (TBR) [5]: It is a multipath QoS routing scheme and QoS resource reservation are done along multipath. This protocol uses tickets to find the feasible path which could be either delay constrained or bandwidth constrained. There are two types of tickets used, one is for finding a route with delay/bandwidth constrained and the other one is for determining low cost routes. If the number of tickets issued by the host is more then there is a better chance of finding a feasible path [6]. Each node is incorporated with resource availability of its neighbor node for resource estimation. As each node must keep complete information of each of its neighbors which requires more memory and this is the main drawback of this protocol. The destination node acknowledges the primary route information to the source node and hence in this protocol resource reservation is established.

- QoS Optimized Link State Routing (QOLSR) [7]: QOLSR protocol is an enhancement of the OLSR routing protocol to support multiple QoS metric in a routing. OLSR is a proactive routing protocol and based on the properties of a link state algorithm and Dijkstra's shortest path algorithm to provide optimal routes in terms of number of hops. The QoS metrics considered in OLSR are throughput and delay. The routes are immediately available when needed. It minimizes the control overhead involved in flooding routing information. In which MAC protocol is required to notify the routing protocol when it transmits a packet. Using the periodic HELLO message delay or residual bandwidth are estimated statistically and it does not rely on MAC protocol.

3) Based on the routing information update mechanism employed:

- Predictive Location Based QoS Routing protocol (PLBQR) [8]: It is based on prediction of the location of nodes in ad hoc wireless networks. The location prediction is used to predict the geographic location of the node at a particular instant of time when the packet reaches that node. Location-resource, update protocol is used to predict the locationdelay of a node. No resources are reserved along the path from source to destination. QoS routing information used location prediction mechanism and resource information for all nodes in the network obtained from the location update mechanism. The advantage of this protocol is the prediction of new location based on previous location is made when there is variation in the geographical location. There are dynamic changes in the direction and hence accurate prediction on velocity and direction is not made. The location prediction is made only in a linear pattern (i.e., the angular velocity is kept at zero).

- $\quad$ Bandwidth Routing protocol (BR) [9]: This protocol informs the source node about available end-to-end bandwidth using bandwidth calculation algorithm. The bandwidth reservation scheme is employed to reserve a sufficient number of free time slots for QoS routing as the wireless channel is time-slotted. It is based on destination sequenced distance vector (DSDV) routing scheme. Bandwidth is calculated by the number of available time slots between source and destination. This protocol uses multiple paths, when the primary path fails; the secondary path is used and hence reduces the packet loss. It provides efficient bandwidth allocation for CDMA over TDMA. The network needs to be fully synchronized because if a particular node leaves the network the corresponding slot remains unused and it cannot be reused.

\section{B. Classification based on the layer}

1) MAC/DLL solutions:

- $\quad$ Cluster TDMA [10]: This solution is proposed by M. Gerla and J.T.C. Tsai in 1995 for supporting real-time traffic in ad hoc wireless networks. In QoS constrained ad-hoc wireless networks, the limited resources available need to be managed. In this clustering scheme, nodes split into different groups. Each group has a cluster-head (elected by members of that group), that act as a regional broadcast node and as a local coordinator to enhance the channel throughput. Every node within a cluster is one hop away from the cluster-head $(\mathrm{CH})$. The formation of clusters and selection of cluster heads is done in a distributed manner. Clustering algorithms split the nodes into clusters in a way that each node is interconnected to every other node. Three such algorithms used are lowest-ID algorithm, highestdegree algorithm, and least cluster change algorithm. These entire clustering algorithms use different method for the 
selection of cluster-head in a cluster. This approach uses fixed clustering scheme, divides the network into fixed size clusters. A node comes under this cluster become part of the cluster or cluster member (CM).

- IEEE 802.11e [11] [12]: IEEE 802.11e provides new coordination function is called Hybrid Coordination Function (HCF). HCF defines two ways for channel access which are HCF Controlled Channel Access (HCCA) is centralbased used in LAN with access points and Enhanced Distributed Channel Access (EDCA) is contention-based and provides differentiated and distributed access to the wireless medium by utilizing four Access Categories i.e. background (0), best effort (1), video (2) and voice (3). EDCA channel access has up to eight AC's. This differentiation is done by varying the duration called Arbitration Inter-Frame Space (AIFS) and Contention Window (CW), where access category (AC) with shorter AIFS and shorter contention window have high priority and vice versa (voice has the highest priority and a background has the lowest priority as voice data is delay sensitive). Once the contenting node detects the medium is idle for AIFS assigned by that AC, it starts a back-off operation by setting a counter to a value is taken randomly from the interval $[0, \mathrm{CW}]$, where $\mathrm{CW}$ is taken between two values are called minimum contention window (CWmin) and maximum contention window (CWmax), those values of contention window are parameters depend on the AC. Each AC has a Transmission Opportunity (TXOP) limiting, a station has right to initiate its transmission during this TXOP. A station may transmit one or more MAC service data unit (MSDU) depending on the duration of TXOP.

\section{2) Network layer solutions}

- $\quad$ Trigger-based Distributed-QoS Routing (TDR) [13]: It is another location based protocol. In this every host node is required to maintain two databases. One is about local neighbors and the other one is activity based. Hosts are required to periodically broadcast beacons that carry their location and mobility information. The mobility and location information are supposed to be transferred by the hosts and upon receiving this, neighbor nodes must record the power level as well as the location and mobility information in their local neighbors database. The routing information of every session is supposed to be recorded in an activity-based. The stable route is selected by forming links with neighboring nodes whose power level of the received packet is greater than the threshold value. This protocol calls admission during route discovery, soft reservations, and route break prediction to support QoS. During TDR route maintenance is done by defining three different receive-power levels, Pth1 > Pth2 > Pcr, to predict route breaks. When the receive-power level at a particular link is lower than Pcr, the upstream active node initiates a rerouting process that is called link degradation triggered rerouting.

\section{3) QoS Framework (cross-layer solutions)}

Framework is a collection of modules employed to provide promised QoS support in MANET. The QoS service model is the main components of any QoS framework, this model defines the way how user requirements are met. The other main components of the framework are QoS routing, QoS medium access control, QoS signalling, packet scheduling schemes and call admission control. QoS models for ad hoc wireless network are described below:

- $\quad$ FQMM [14]: The flexible QoS model for mobile ad hoc networks (FQMM) is the first QoS model which is a hybrid combination of both integrated services (IntServ) model and differentiated services (DiffServ) model. Salient features of FQMM include: dynamics roles of nodes, hybrid provisioning and adaptive conditioning. This model solves the problem of scalability and act as a base for other QoS models, but suffers from the problems such as traffic classification and scheduling.

- INSIGNIA (In-band Signalling System for Supporting QoS in Mobile Ad Hoc Networks) [15]: It is the first signalling protocol designed solely for MANETs. It is characterized as an "In-band RSVP" protocol. It encapsulates control info in the IP Option field (called now INSIGNIA Option field). It keeps flowing state for the real time (RT) flows. It is "Soft State" model. Reservation Mode (REQ/RES) indicates whether there is already a reservation for this packet. If "no", the packet is forwarded to INSIGNIA Module, which in coordination with an AC (access categories) may either RT (real-time) or BE (best-effort). If "yes", the packet will be forwarded with the allowed resources. Bandwidth Request (MAX/MIN) indicates the requested amount of bandwidth.

- $\quad$ SWAN [16]: Stateless Wireless Ad hoc Networks (SWAN) is a distributed network model offers a stateless QoS model for MANETs. It uses traffic differentiation which differentiates real- time and best effort traffic and shapes only the best-effort packets using a leaky bucket traffic shaper. Bandwidth estimation is done by sending a probe message to the destination. It is stateless model and hence there is no need to maintain state in the intermediate nodes from source to destination. In SWAN, ECN (explicit congestion notification) is used for dynamically regulating admitted real-time sessions. The advantage of this model, it makes the system scalable, simple, and robust and solves the problem of false admission, but increases the jitter and latency for real time flows and suffers from high signalling 
Table 1: Classification of QoS solutions based on the QoS metrics

\begin{tabular}{|c|c|c|}
\hline Number of metrics & Metrics & QoS solution \\
\hline \multirow{10}{*}{ Single } & \multirow{8}{*}{ Bandwidth } & INSIGNIA [15] \\
\hline & & CEDAR [3] \\
\hline & & TDR [13] \\
\hline & & $\mathrm{BR}[9]$ \\
\hline & & OQR [20] \\
\hline & & $\mathrm{MRP}[21]$ \\
\hline & & QoS-TORA [22] \\
\hline & & INORA [23] \\
\hline & \multirow[b]{2}{*}{ Power } & PAMAS [17] \\
\hline & & CMMBCR [18] \\
\hline \multirow{6}{*}{ Double } & \multirow{4}{*}{ Bandwidth and Delay } & QAODV [4] \\
\hline & & TBP [5] \\
\hline & & PLBQR [8] \\
\hline & & AQOR [24] \\
\hline & Throughput and Delay & QOLSR [7] \\
\hline & $\begin{array}{l}\text { Bounded delay and } \\
\text { Packet loss rate }\end{array}$ & GAMAN [25] \\
\hline Multiple & Delay, Jitter and Energy & SDVR [19] \\
\hline
\end{tabular}

\section{Classification based on the QoS metrics}

1) QoS routing protocol with single metric

QoS routing protocol with single QoS constraint is mentioned in Table 1. These protocols take only single QoS parameters into consideration. Some of them are listed below in table 2 and discussed in previous sections. The QoS metric of a Power Aware Multiple Access Protocol (PAMAS) [17] is power. When a node knows that it will not be able to send and receive packets during specific duration, it will turn off its radio interface during that time because of the possibility of multiple access interference. In this algorithm sleep time of a node is of the order of packet duration. Conditional Max-Min Battery Capacity Routing (CMMBCR) [18] is also single metric and its QoS metric is power. This algorithm selects the route on the basis of minimal total transmission power if all the nodes in the route have residual battery capacities higher than a threshold and routes with nodes having lowest residual battery capacities are avoided. A route with minimum total transmission power among multiple and having enough residual energy is selected. This algorithm considers the residual energy of nodes and total transmission energy utilization of routes.

\section{2) QoS routing protocol with double metrics}

Some of the QoS solutions with double QoS metrics are mentioned in Table 1 with QoS parameter bandwidth and delay or throughput and delay or delay and packet loss rate. These solutions are explained in the previous sections.

3) QoS routing protocol with multiple metrics

- SDVR [19]: A Swarm-based Distance Vector Routing optimizes three QoS parameters delay, jitter and energy. The mechanism was based on information obtained from periodically transmitted backward ANTs. This routing protocol selects a path of the maximum residual energy at nodes and minimum delay. It also takes jitter as a QoS parameter into consideration in order to keep the minimum and maximum delay values approximate the average delay. SDVR results are better than well known MANET routing protocol, i.e. AODV in terms of end-to-end delay, packet delivery ratio and residual energy but its overhead are high as compared to AODV.

\section{QoS routing protocol with optimization techniques}

1) QoS routing based on genetic algorithm optimization technique

- GAMAN [25]: A Genetic Algorithm (GA) based routing method for Mobile Ad hoc Networks (GAMAN) is proposed. This algorithm takes delay and packet loss rate as QoS constraints. In this solution genetic algorithm is used to find the optimal route heuristically based on the QoS constraints mentioned. Reliability and delay are taken as a fitness 
value to select paths for crossover and mutation operation. Small population size employed only few nodes are involved in route computation. The dissemination of information is limited by transmitted to only nodes in a population. This is multipath routing as GA explores different routes and they are ranked by sorting. The first route is the best route and the other routes are used as backup routes.

Table 2: Summarization of some frequently used QoS solutions.

\begin{tabular}{|c|c|c|c|c|c|c|}
\hline $\begin{array}{c}\text { QoS Routing } \\
\text { Protocol }\end{array}$ & $\begin{array}{c}\text { QoS } \\
\text { Metrics }\end{array}$ & $\begin{array}{c}\text { Network } \\
\text { Architecture }\end{array}$ & $\begin{array}{c}\text { Route } \\
\text { Discovery }\end{array}$ & $\begin{array}{c}\text { Resource } \\
\text { Reservation }\end{array}$ & $\begin{array}{c}\text { Multiple } \\
\text { paths }\end{array}$ & $\begin{array}{c}\text { QoS } \\
\text { Guarantee }\end{array}$ \\
\hline CEDAR [3] & Bandwidth & Hierarchical & $\begin{array}{c}\text { Proactive/ } \\
\text { Reactive }\end{array}$ & Yes & No & Soft \\
\hline QAODV [4] & Bandwidth, Delay & Flat & Reactive & No & No & Soft \\
\hline TBP [5] & Bandwidth, Delay & Flat & Reactive & Yes & Soft \\
\hline QOLSR [7] & $\begin{array}{c}\text { Throughput and } \\
\text { Delay }\end{array}$ & Hierarchical & Proactive & No & Soft \\
\hline PLBQR [8] & $\begin{array}{c}\text { Bandwidth, Delay } \\
\text { BR [9] }\end{array}$ & $\begin{array}{c}\text { Location } \\
\text { prediction }\end{array}$ & Proactive & No & Soft \\
\hline TDR [13] & Bandwidth & Focation & Reactive & Yes & No & Soft \\
\hline INSIGNIA [15] & Bandwidth & Flat & Reactive & Yes & No & Soft \\
\hline GAMAN [25] & $\begin{array}{c}\text { Bounded delay, } \\
\text { Packet loss rate }\end{array}$ & Hierarchical & Reactive & Yes & Yes & Soft \\
\hline
\end{tabular}

2) QoS routing based on ant-colony based optimization technique

- ARA [26]: The Ant colony based Routing Algorithm (ARA) is based on both swarm intelligence and ant-colony optimization algorithm. It consists of three phases: route discovery, route maintenance, and route break handling. New routes between nodes are discovered in the route discovery phase, with the use of forward and backward ants. Routes are maintained by subsequent data packets by node pheromone values.

\section{COMPARISON OF QOS SOLUTIONS FOR MANET}

Comparison of some well known and frequently used QoS solutions on the basis of their QoS metrics, type of network architecture, route discovery, resource reservation and redundant paths is summarized as shows that in Table 2. Some of the discussed MANET QoS routing protocols in this paper are summarized in the Table 2. Selection of any routing protocol based on the requirement of the application can be easily done from this table.

\section{CONCLUSION}

Providing QoS Support in mobile ad hoc networks is one of the interesting research areas and the unique characteristics of MANETs makes several difficulties in QoS provisioning. Critical and detailed analysis of various types of QoS solutions for mobile ad hoc networks is done. This comparision are summarized in Table 1 and Table 2 on the basis of its attributes and characteristics and various critical parameters of adhoc networks. A lot of research has been done in the field of QoS aware routing protocol. This fundamental work is useful for new researchers to identify the critical parameter and scope for improvement and invention of new QoS aware routing protocol in MANET for achieving particular objective.

\section{REFERENCES}

[1] R. B. Ali and S. Pierre, "An Efficient Predictive Admission Control Policy for Heterogeneous Wireless Bandwidth Allocation in Next Generation Mobile Networks", IEEE Transaction Networking 1997, Vol. 5(1), pp.1-12.

[2] J.F. Kurose, M. Schwartz, and Y.Yemini, Multiple access protocols and time constraint communications, ACM Computing Surveys, Vol. 16, pp. 43-70, 1984.

[3] R.Sivakumar, P.Sinha and V.Bharghavan, "CEDAR: A Core-Extraction Distributed Ad Hoc Routing Algorithm," IEEE Journal on Selected Areas in Commusnications, vol.17, no. 8, pp.1454-1465, August 1999.

[4] C. E. Perkins, E. M. Royer and S. R. Das, "Quality of Service for Ad Hoc On Demand Distance Vector (AODV) Routing," IETF Internet-Draft, draft-ietf-manet-aodvqos-00.txt, July 2000.

[5] S. Chen and K. Nahrstedt, "Distributed Quality-of-Service in Ad Hoc Networks," IEEE JSAC, vol. 17, no. 8, 1999. 
[6] Lei Chen and Wendi B. Heinzelman, "A Survey of Routing Protocols that Support QoS in Mobile Ad Hoc Networks", IEEE Network November/December 2007.

[7] Y. Ge, T. Kunz, and L. Lamont, "Quality of Service Routing in Ad-Hoc Networks Using OLSR," Proc. 36th Hawaii International Conference Sys. Sci., Jan. 2003.

[8] S.H.Shah and K.Nahrstedt, "Predictive Location- Based QoS Routing in Mobile Ad Hoc Networks," Proceedings of IEEE ICC 2002, vol.2, pp.1022-1027, May 2002.

[9] Lin CR, Liu J-S. "QoS routing in ad hoc wireless networks" IEEE Journal on Selected Areas in Communications, vol. 17, no.8, pp. 1426-38, August 1999.

[10] M. Gerla and J. T. C. Tsai, "Multicluster, Mobile, Multimedia Radio Network," ACM/Baltzar Wireless Networks Journal, vol. 1, no. 3, pp. 255-265, October 1995.

[11] Y. Chen, Q. Zeng, and D.P. Agrawal, "Performance evaluation for IEEE 802.11 e enhanced distributed coordination function," Wireless Communications and Mobile Computing, 2004, vol. 4,pp. 639-653.

[12] D. Gu and J. Zhang, "QoS enhancement in IEEE S02.11 wireless local area networks," IEEE Communication Mag., pp. 120-124, June 2003.

[13] S. De, S. K. Das, H. Wu, and C. Qiao, "Trigger-Based Distributed QoS Routing in Mobile Ad Hoc Networks," ACM SIGMOBILE Mobile Computing and Communication Review, vol. 6, no. 3, pp. 22-35, July 2002.

[14] H. XIAO, Winston K.G. SEAH, Anthony LO and Kee Chiang CHUA “Flexible Quality of Service Model for Mobile AdHOC Networks, " In proceedings of the IEEE Vehicular Technology Conference, May 2000.

[15] S. B. Lee, A. Gahng-Seop, X. Zhang, and A. T. Campbell, "INSIGNIA: An IP-Based Quality of Service Framework for Mobile Ad Hoc Networks," Journal of parallel and Distributed Computing, vol. 60, no.4, pp.374-406, April 2000.

[16] H. Ahn, A T. Campbell, A. Veres, and L.Sun, "Supporting Service Differentiation for Real-Time and Best-Effort Traffic in Stateless Wireless Ad Hoc Networks," IEEE transaction on mobile computing, vol. 1, no. 3, pp. 192-207, September 2002.

[17] S. Singh. and C.S Raghavendra, "PAMAS - Power Aware Multi Access protocol with Signaling for ad hoc networks" ACM Computer Communication Review, Vol. 28 No. 3, pp. 5-26, July 1998.

[18] C.K Toh, "Maximum battery life routing to support ubiquitous mobile computing in wireless ad hoc networks" IEEE Communication Magazine, Vol. 39, no. 6, pp. 138 - 147, June 2001.

[19] R. Asokan, A.M Natarajan and A.Nivetha, "A Swarm-based Distance Vector Routing to Support Multiple Quality of Service (QoS) Metrics in Mobile Ad hoc Networks" Journal of Computer Science, Vol.3, No.9, pp.700-707, 2007.

[20] C. R. Lin, "On-Demand QoS Routing in Multi-Hop Mobile Networks," proceeding of IEEE INFOCOM 2001, vol. 3, pp. 1735-1744, April 2001.

[21] W.H Liao, Y.C Tseng, S.L Wang, and J.P Sheu, "A multi-path QoS routing protocol in a wireless mobile ad hoc network" Proceedings of the IEEE International Conference on Networking, Vol. 2, pp. 158-167, 2001

[22] I. Gerasimov, and R. Simon, "A bandwidth reservation mechanism for on-demand ad hoc path finding" Proceedings of the IEEE/SCS $35^{\text {th }}$ Annual Simulation Symposium, San Diego, CA, pp. 27-34, April 2002.

[23] D. Dharmaraju, A. R. Chowdhury, P. Hovareshti, and J.S. Baras , "INORA-A Unified Signalling and Routing Mechanism for QoS Support in Mobile Ad Hoc Networks," Proceedings of ICPPW 2002, pp. 86-93, August 2002.

[24] Q. Xue and A. Ganz, "Ad hoc QoS on-demand routing (AQOR) in Mobile AdHoc Networks," Journal of Parallel and Distributed Computing, vol. 63 no. 2, pp. 154-65, Feb. 2003.

[25] L. Barolli , A. Koyama, T. Suganuma, and N. Shiratori, "GAMAN: A GA based QoS routing method for mobile ad hoc networks" Journal of Interconnection Networks, Vol. 4, no. 3, pp.251-270, 2003.

[26] M. Gunes , U. Sorges, and I. Bouazizi, "ARA - The ant-colony based routing algorithm for MANETs" Proceedings of the International Conference on Parallel Processing Workshops, pp.79-85, August 2002. 